Ferrata Storti Foundation

\title{
Impaired microRNA processing in neutrophils from rheumatoid arthritis patients confers their pathogenic profile. Modulation by biological therapies
}

Haematologica 2020

Volume 105(9):2250-2261

\section{Correspondence:}

NURIA BARBARROJA

nuria.barbarroja.exts@juntadeandalucia.es

Received: August 22, 2018.

Accepted: January 10, 2020.

Pre-published: January 16, 2020.

doi:10.3324/haematol.2018.205047

(C)2020 Ferrata Storti Foundation

Material published in Haematologica is covered by copyright. All rights are reserved to the Ferrata Storti Foundation. Use of published material is allowed under the following terms and conditions:

https://creativecommons.org/licenses/by-nc/4.0/legalcode. Copies of published material are allowed for personal or internal use. Sharing published material for non-commercial purposes is subject to the following conditions:

https://creativecommons.org/licenses/by-nc/4.0/legalcode, sect. 3. Reproducing and sharing published material for commercial purposes is not allowed without permission in writing from the publisher.
Ivan Arias de la Rosa, ${ }^{1 *}$ Carlos Perez-Sanchez, ${ }^{2 *}$ Patricia Ruiz-Limon, ${ }^{3 *}$ Alejandra Patiño-Trives, ${ }^{1}$ Carmen Torres-Granados, ${ }^{1}$ Yolanda Jimenez-Gomez, ${ }^{1}$ Maria del Carmen Abalos-Aguilera, ${ }^{1}$ Irene Cecchi, ${ }^{4}$ Rafaela Ortega, ${ }^{1}$ Miguel Angel Caracuel, ${ }^{1}$ Jerusalem Calvo-Gutierrez, ${ }^{1}$ Alejandro EscuderoContreras, ${ }^{1}$ Eduardo Collantes-Estevez, ${ }^{1 \#}$ Chary Lopez-Pedrera ${ }^{1 *}$ and Nuria Barbarroja $\mathbf{1}^{\# 1,5}$

*IAR, CPS and PRL contributed equally as co-first authors.

\#ECE, CLP and NB contributed equally as co-senior authors.

${ }^{1}$ Rheumatology service, Maimonides Institute for Research in Biomedicine of Cordoba (IMIBIC)/Reina Sofia Hospital/University of Cordoba, Cordoba, Spain; ${ }^{2}$ Deparment of Medicine, University of Cambridge, School of Clinical Medicine, Addenbroke's Hospital, Cambridge Institute for Medical Research, Cambridge, UK; ${ }^{3}$ Biomedical Research Institute (IBIMA), Service of Endocrinology and Nutrition, Malaga Hospital Complex (Virgen de la Victoria), Malaga, Spain; ${ }^{4}$ Department of Clinical and Biological Sciences, Center of Research of Immunopathology and Rare Diseases-Coordinating Center of Piemonte and Valle d'Aosta Network for Rare Diseases, Turin, Italy and ${ }^{5}$ CIBER Fisiopatología de la Obesidad y Nutrición (CIBEROBN), Instituto de Salud Carlos III, Madrid, Spain

\section{ABSTRACT}

T The aim of this study was to investigate the microRNA (miRNA) expression pattern in neutrophils from rheumatoid arthritis (RA) patients and its contribution to their pathogenic profile and to analyze the effect of specific autoantibodies or inflammatory components in the regulation of miRNA in RA neutrophils and its modulation by biological therapies. Neutrophils were isolated from paired peripheral blood (PB) and synovial fluid samples of 40 patients with RA and from $\mathrm{PB}$ of 40 healthy donors. A miRNA array was performed using nCounter technology. Neutrophils from healthy donors were treated in vitro with antibodies to citrullinated protein antigens isolated from RA patients and tumor necrosis factor- $\alpha$ (TNF- $\alpha$ ) or interleukin- 6 . A number of cytokines and chemokines were analyzed. In vitro treatments of RA-neutrophils with tocilizumab or infliximab were carried out. Transfections with pre-miRNA and DICER downregulation experiments were further performed. RA-neutrophils showed a global downregulation of miRNA and genes involved in their biogenesis, alongside with an upregulation of various potential mRNA targets related to migration and inflammation. Decreased levels of miRNA and DICER correlated with autoimmunity, inflammation and disease activity. Citrullinated protein antigens and TNF- $\alpha$ decreased the expression of numerous miRNA and their biogenesis-related genes, increasing their potential mRNA targets. Infliximab reversed those effects. Transfections with pre-miRNA-223, -126 and -148a specifically modulated genes regulating inflammation, survival and migration whereas DICER depletion influenced the inflammatory profile of neutrophils. Taken together RA-neutrophils exhibited a global low abundance of miRNA induced by autoantibodies and inflammatory markers, which potentially contributed to their pathogenic activation. miRNA biogenesis was significantly impaired in RAneutrophils and further associated with a greater downregulation of miRNA mainly related to migration and inflammation in synovial fluid neutrophils. Finally, anti-TNF- $\alpha$ and anti-interleukin- 6 receptor treatments can modulate miRNA levels in the neutrophils, minimizing their inflammatory profile. 


\section{Introduction}

Several immune cells including $\mathrm{T}$ and $\mathrm{B}$ lymphocytes, macrophages, synovial fluid (SF) fibroblast and neutrophils are known to be relevant in the rheumatoid arthritis (RA) pathogenesis. ${ }^{1}$ Among them, RA neutrophils are activated cells, characterized by a prolonged lifespam, increased migratory capacity and production of inflammatory molecules and reactive oxygen species (ROS). In severe acute inflammation, SF accumulates a great number of these cells in a more activated state, promoting cartilage destruction and joint damage. ${ }^{2}$

Antibodies to citrullinated protein antigens (ACPA) are currently considered the most specific autoantibodies in RA, being related to the activity of the disease and poorer prognosis. ${ }^{3}$ ACPA have been shown to be able to induce neutrophils to produce high levels of inflammatory mediators, ROS and to generate NETosis.,4

Epigenetic modifications contribute to the development of RA, affecting disease susceptibility and severity, ${ }^{5,6}$ Among them, several microRNA (miRNA) have been linked to the chronic inflammation in RA. ${ }^{5}$ MiRNA are short noncoding RNA present in all multi-cellular organisms involved in a broad range of cellular processes. They cause posttranscriptional and posttranslational gene silencing, by recognizing a specific sequence of mRNA, binding to it and inhibiting its translation into protein. MiRNA is first transcribed into long primary miRNA of several $\mathrm{kb}$ in length (pri-miRNA) and this pri-miRNA is then processed by Drosha into a precusor miRNA (premiRNA) of appoximately 70-nucleotide. The pre-miRNA is transported out of the nucleus by exportin 5 (XPO-5) and is then processed by DICER into a mature double stranded miRNA of approximately 22 nucleotides. The RNA-induced silencing complex (RISC) (composed of the transactivation-responsive RNA-binding protein [TRBP] and argonaute [AGO]) removes the complementary strand. DICER then binds to RISC, forming the core of RISC-loading complex. DICER is considered a crucial factor in miRNA processing since its presence is necessary for the stimulation of RNA processing by AGO ${ }^{8,9}$ Functional miRNA is able to bind to the 3 '-untranslated region (UTR) of the target mRNA, causing mRNA cleavage or translational repression. ${ }^{10}$

Several studies, mainly conducted on lymphocytes, monocytes, macrophages and SF fibroblasts, have reported that the role of various miRNAs in the pathogenesis of $\mathrm{RA}$ is critical for the increased expression of inflammatory cytokines and prolonged cell survival..$^{5,11}$

We undertook this study to evaluate the miRNA profile and the proteins involved in miRNA processing in circulating and SF neutrophils from RA patients, in order to gain an insight of its role in the different activation states of these cells. The effects of ACPA or inflammatory components and biological therapies on the expression of miRNA in neutrophils was further assessed.

\section{Methods}

For details see the Online Supplemental Materials and Methods.

\section{RA patients and healthy donors}

Forty RA patients and 40 healthy donors (HD) were included in this study. RA patients fulfilled at least four 1987 American College of Rheumatology (ACR) criteria and achieved a total score of 6 or greater according to 2010 criteria. The patients were under the following treatment regimes: corticosteroids (50.0\%), leflunomide $(42.5 \%)$, hydroxychloroquine $(45.0 \%)$, NSAID $(80.0 \%)$ and methotrexate (65\%). All patients were tested for the presence of ACPA and rheumatoid factor (RF) by clinical laboratory routine analysis. All participants enrolled were Caucasian, recruited at the Department of Rheumatology, and gave their written informed consent approved by the ethical committee of the Reina Sofia Hospital (Cordoba, Spain).

Clinical details of the RA patients and $\mathrm{HD}$ are shown in Table 1. Peripheral blood (PB) was withdrawn from all the RA patients and the HD. SF from RA patients was obtained through arthrocentesis. The study design is displayed on a flow chart (Online Supplementary Figure S1).

\section{Isolation of neutrophils from PB and SF}

Neutrophils from $\mathrm{PB}$ of $\mathrm{HD}$ and paired $\mathrm{SF}$ and $\mathrm{PB}$ samples of $\mathrm{RA}$ patients were isolated (after centrifugation to obtain buffy coat and osmotic lysis of the pellet) by immunomagnetic positive selection with human anti-CD15 microbeads (Miltenyi Biotec S.L, Bergisch Gladbach, Germany) using AUTOMACs (Miltenyi Biotec). ${ }^{12}$

\section{miRNA expression profiling}

The nCounter miRNA Assay (NanoString Technology, Seattle, WA, USA) detects simultaneously 800 human miRNA in each sample. 100 ng of RNA, pooled samples of neutrophils from PB of $10 \mathrm{HD}$, neutrophils from $\mathrm{PB}$ of $10 \mathrm{RA}$ patients and neutrophils from SF of 10 RA patients were prepared by ligating a specific DNA tag (miR-tag) onto the 3 end of each mature miRNA followed by $16-20$ hour hybridization (at $65^{\circ} \mathrm{C}$ ) to nCounter Reporter and Capture probes. The rest of the protocol was performed following the manufacturer's recommendations (NanoString Technologies, Seattle, WA, USA). Data were normalized by the geometric mean of top 100 miRNA detected using the nSolver software. This miRNA array was performed in pooled samples of the 10 RA patients that best represented the mean values of age, sex, disease activity, evolution time and autoimmunity of the clinical validation cohort.

\section{IgG-ACPA isolation from RA patients}

Immunoglobulin G (IgG) from serum of five different RA patients with high titers of ACPA and negative for RF (enriched IgG-ACPA) and 5 HD (IgG- normal human serum [NHS]) were isolated using HiTrap protein G HP columns (GE Healthcare, Chicago, Il, USA).

\section{In vitro treatments of neutrophils}

Neutrophils purified from five RA patients (taking Diseasemodifying antirheumatic drugs and not taking any biological therapies) were pre-treated with FCRII blocking reagent (Miltenyi Biotec, Bergisch Gladbach, Germany) for $15 \mathrm{~min}$ and subsequently incubated with infliximab (IFX) at $100 \mathrm{~g} / \mathrm{mL}$ or tocilizumab (TCZ) at $20 \mathrm{~g} / \mathrm{mL}$ for 6 hours. The selection of these patients allowed the isolation of assumingly activated neutrophils, leading to an increased expression of inflammatory cytokines, in order to demonstrate the effects of miRNA transfection by proving the reduction in expression levels of those molecules.

Neutrophils purified from five $\mathrm{HD}$ were treated in vitro with IgG-NHS or enriched IgG-ACPA (500 ug/mL), tumor necrosis factor- $\alpha$ (TNF- $\alpha)$ and interleukin- 6 (IL- $\sigma)(10 \mathrm{ng} / \mathrm{mL})$ for 6 hours Samples were processed for RT-PCR analyses. 
Table 1. Clinical details of rheumatoid arthritis patients and healthy donors.

\begin{tabular}{|c|c|c|c|}
\hline Clinical parameters & RA patients ( $n=40)$ & HD controls ( $n=40)$ & $P$ \\
\hline Woman/Men (n/n) & $26 / 14$ & $22 / 18$ & \\
\hline Age (years) & $55.54 \pm 4.03$ & $48.50 \pm 8.45$ & \\
\hline Disease evolution (years) & $9.20 \pm 2.23$ & - & \\
\hline $\mathrm{RF}^{+}(\%)$ & 57.50 & - & \\
\hline $\mathrm{ACPA}^{+}(\%)$ & 65.00 & - & \\
\hline Obesity (\%) & 8.00 & 3.00 & 0.035 \\
\hline Diabetes (\%) & 10.00 & 5.00 & 0.033 \\
\hline Hypertension (\%) & 12.50 & 6.00 & 0.048 \\
\hline Smoker (\%) & 15.00 & 12.00 & \\
\hline DAS28 & $3.75 \pm 0.20$ & - & \\
\hline Cholesterol (mg/mL) & $202.15 \pm 12.65$ & $200.20 \pm 15.60$ & \\
\hline HDL-Cholesterol (mg/mL) & $43.16 \pm 2.68$ & $53.63 \pm 12.75$ & \\
\hline LDL-Cholesterol (mg/mL) & $136.81 \pm 11.01$ & $115.75 \pm 17.40$ & \\
\hline Triglycerides (mg/mL) & $99.50 \pm 10.16$ & $88.50 \pm 25.39$ & \\
\hline $\operatorname{ESR}(\mathrm{mm} / \mathrm{h})$ & $15.75 \pm 2.89$ & $4.65 \pm 1.20$ & 0.01 \\
\hline $\mathrm{CRP}(\mathrm{mg} / \mathrm{mL})$ & $10.23 \pm 2.73$ & $1.55 \pm 0.22$ & 0.03 \\
\hline \multicolumn{4}{|l|}{ Treatments } \\
\hline Corticosteroids (\%) & 50.00 & - & \\
\hline Hydroxychloroquine (\%) & 45.00 & - & \\
\hline NSAIDs (\%) & 80.00 & - & \\
\hline Methotrexate (\%) & 70.00 & - & \\
\hline
\end{tabular}

\section{Results}

\section{Global decrease in miRNA levels of neutrophils from RA patients}

Among the 800 miRNA analyzed, levels of 133 miRNA were detected in neutrophils. Using an above two-fold change cut-off, 94 miRNA were reduced in PB-RA neutrophils compared to PB-HD, and three of them were elevated (Online Supplementary Table S1 and Figure 1A). Additionally, SF neutrophils showed 34 miRNA even further reduced compared to its paired PB sample (fold change cut-off change above two-fold) (Figure 1B). Ingenuity Pathway Analysis (OIAGEN IPA) uncovered the main enriched biological functions and pathways in which these miRNA are involved, which include immune disease, inflammatory response and connective disorders (Figure 1D).

\section{Low abundance of miRNA levels in RA neutrophils might be due to a defect in the miRNA processing}

Eight altered miRNA were identified by OIAGEN IPA as the main regulators of proteins involved in the abnormal activation of neutrophils in RA, including miRNA -126, 148a, -29c, let-7b, -30c, -17, -21 and 223 (Figure 2). The expression of these miRNA was validated in all the samples separately and a technical validation was performed separately in the 10 samples previously used for the pool. In addition, a clinical validation was carried out separately in the 30 remaining samples (Online Supplementary Figure S2). The levels of most of the selected miRNA were significantly reduced in PB-RA neutrophils compared to PB-HD neutrophils. A greater reduction in the expression of miR148a, miR-29c and let-7b in the SF paired samples was observed (Figure 3A). In addition, there was not significant difference in the reduced levels of miRNA among patients treated or not treated with methotrexate (Online Supplementary Figure S3).

There was a significant reduction in the expression of genes involved in the miRNA processing (DICER and AGO-1) in neutrophils from PB-RA patients compared to PB-HD. Of note, DICER, AGO-1, AGO-2 and XPO-5 were diminished in neutrophils from the SF of RA patients (Figure 3B).

\section{Bioinformatic identification and expression of the putative targets of reduced miRNA in RA neutrophils}

Seven putative mRNA targets were chosen based on their recognized role in the pathogenesis of RA, being key factors in inflammation (TNF- $\alpha$, IL-1 $\beta$, IL-6R), cell adhesion (VEGF-A), migration (IL-8) and survival (STAT3 and AKT). These targets were significantly upregulated in PBRA neutrophils (Figure 3C). A greater alteration was observed in SF neutrophils.

Using enrichment analysis of those selected targets, enriched pathways mainly related to inflammatory processes were revealed. This included a broad range of secondary chemokines and cytokines which are indirectly connected with the eight selected mRNA targets, amplifying the inflammatory cascade (Online Supplementary Figure S4). Thus, a human cytokine array was performed in neutrophils from RA patients (PB and $\mathrm{SF})$ and $\mathrm{HD}(\mathrm{PB})$.

Neutrophils from $\mathrm{PB}$ of RA patients showed increased protein expression of CCL5, CD40L, CXCL1, CXCL2, IL1ra, IL-16, IL-18, IL-32a, PAI-1 and TREM-1 compared to HD (Figure 3D). A differential proteome profile was observed in neutrophils from SF of RA patients compared 

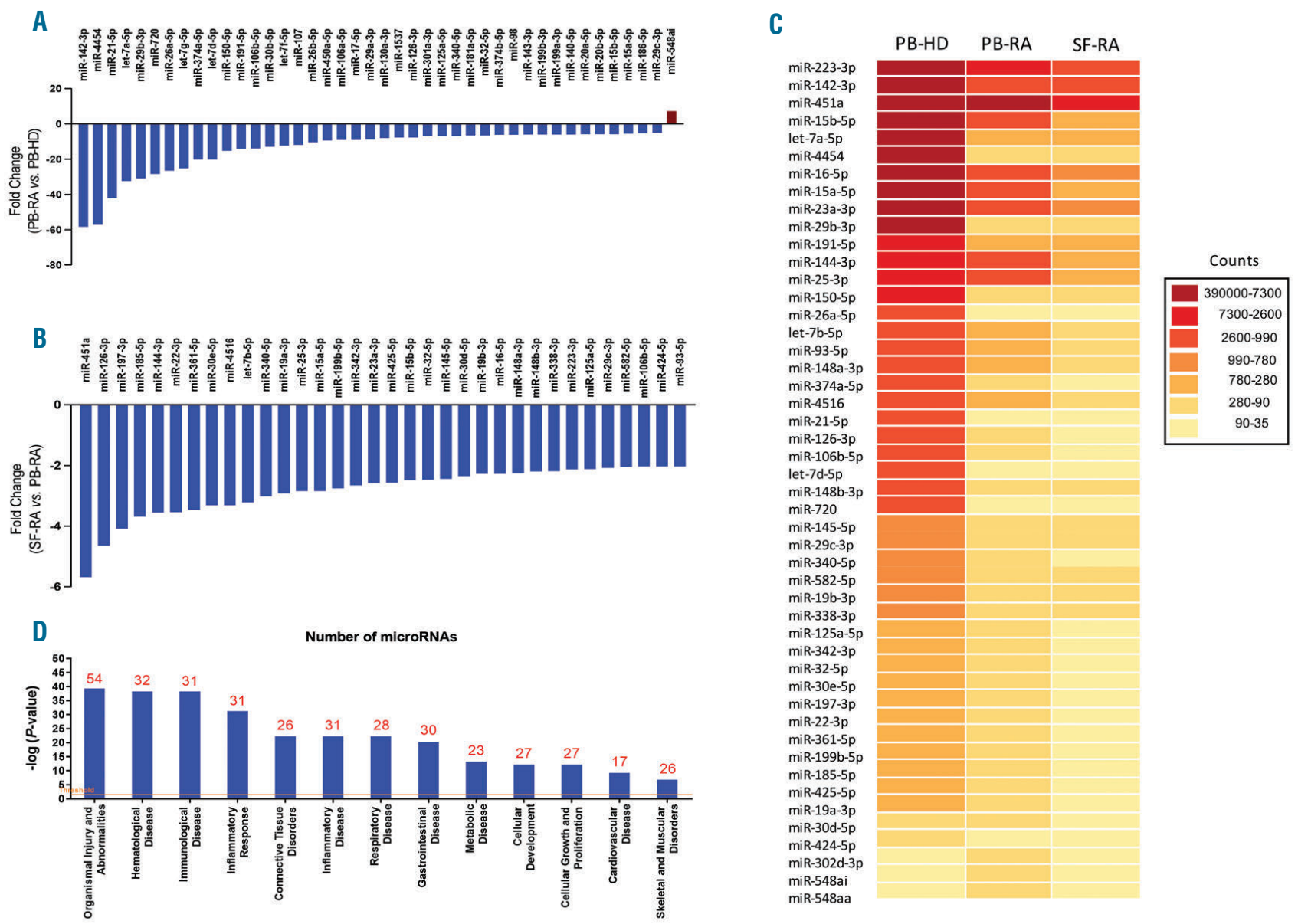

miR-548aa

Figure 1. Global downregulation of miRNA expression profile in rheumatoid arthritis neutrophils. (A) Forty-one miRNA altered in neutrophils from PB-RA patients compared to neutrophils from PB-HD using a fold change cut-off $>5$. (B) Thirty-four miRNA more reduced in RA synovial neutrophils compared to its paired PB sample (fold change cut-off >2). (C) Heat-map of the differentially expressed miRNA profile in PB-HD, PB-RA and SF-RA. (D) Functional classification of the altered miRNA using Ingenuity Pathway Analysis (IPA, QIAGEN Redwood City, CA, USA; https://analysis.ingenuity.com). The analysis included only the functions and pathways with average IPA score $>2$ indicated as - $\log (P$-value). Threshold bar indicates the cut-off point of significance $(P>0.05)$, using Fisher's exact test. miRNA: microRNA; RA: rheumatoid arthritis; PB: peripheral blood; HD: healthy donor; SF: synovial fluid.

to $\mathrm{HD}$ and $\mathrm{PB}$ paired samples (MIP-1/1, CCL5, CD40L, C5/5a, CXCL1, CXCL12, ICAM-1, IL-1, IL-1ra, IL-8, IL13, IL-16, IL-18, MIF and TREM-1) (Figure 3D).

\section{Reduced levels of miRNA in RA neutrophils are related} to autoimmunity, clinical and serological parameters

Decreased levels of both, miRNA and DICER significantly correlated with the activity of the disease, levels of ACPA and clinical inflammatory markers. Elevated serum levels of TNF- $\alpha$ correlated with low levels of DICER. However, there was not association between the levels of miRNA and serum TNF- $\alpha$ (Figure 4A).

\section{ACPA reduces miRNA levels in healthy neutrophils}

Enriched IgG-ACPA downregulated the expression of the eight selected miRNA in healthy neutrophils (Figure 4B). Accordingly, a significant reduction of genes involved in the miRNA biogenesis was observed (Figure 4C). Enriched IgG-ACPA also increased the expression of the selected mRNA targets (Figure 4D). Finally, enriched IgGACPA promoted a significant upregulation of secondary chemokines and cytokines indirectly related with the eight selected mRNA targets (CCL1, CCL2, MIP-1 $\alpha / \beta$, CCL5, CD40L, CXCL1, CXCL12, G-CSF, GM-CSF, IFN- $\gamma$ and IL-8) (Figure 4E)

Inflammatory mediators decrease the expression of miRNA in neutrophils, which might be restored by IFX or TCZ

TNF- $\alpha$ and IL- 6 levels were significant elevated in serum from RA patients; a further increase was observed in SF from those RA patients (Figure 5A).

In vitro, TNF- $\alpha$ downregulated the levels of the eight selected miRNA alongside with a decrease in the expression of DICER and AGO-2 in HD neutrophils (Figure 5B). Treatment with IL- 6 reduced the levels of miR-126, let-7b, miR-17, AGO1 and AGO2 (Figure 5C).

We observed that fresh neutrophils from RA patients had significantly higher levels of TNF- $\alpha$ and IL- 6 mRNA compared to freshly isolated neutrophils from HD (Online Supplementary Figure S5A). In addition, after 6 hours of in vitro culture, levels of TNF- $\alpha$ and IL- 6 were elevated in the culture media of RA neutrophils (Online Supplementary Figure $S 5 B$ ). 


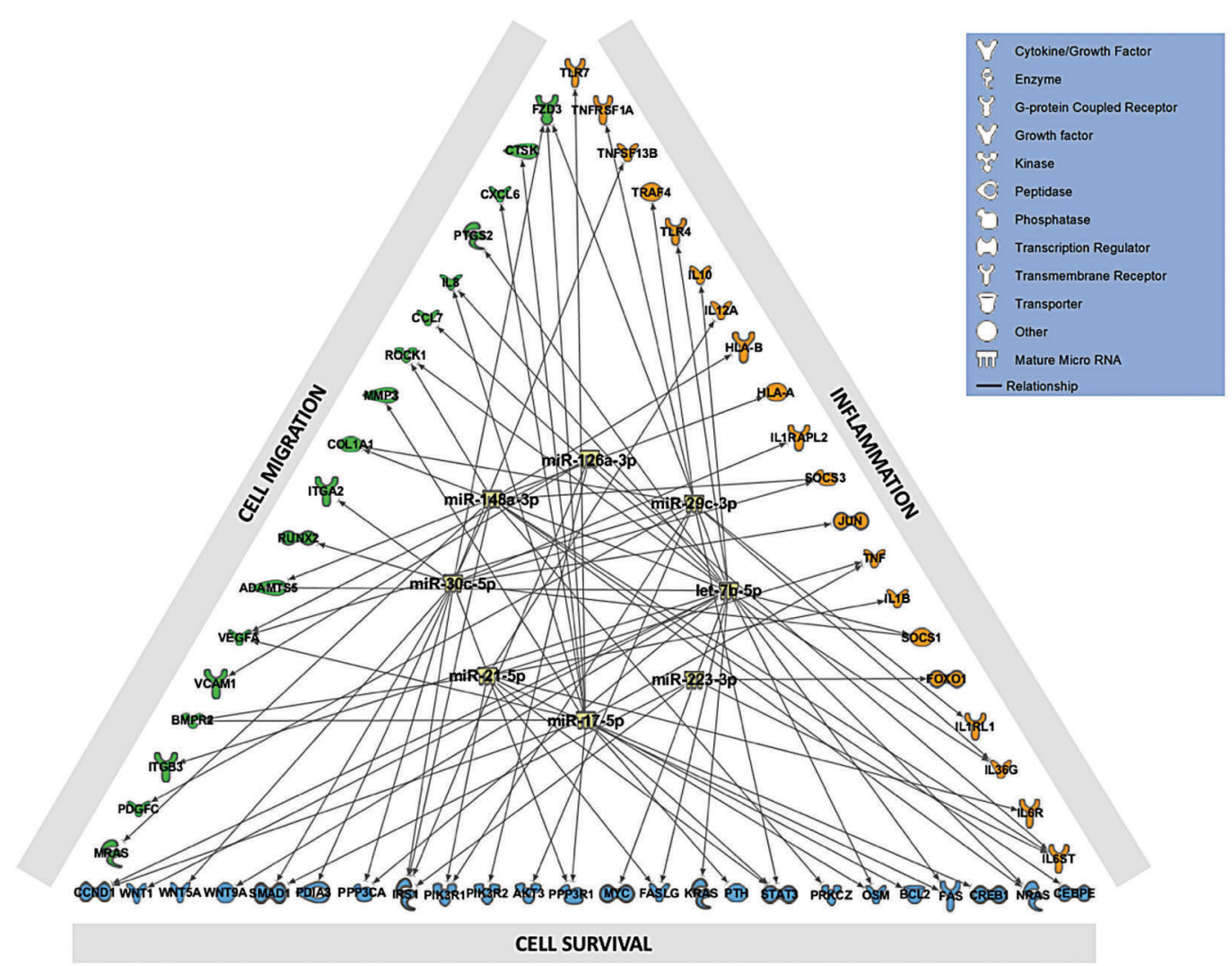

Figure 2. Bioinformatic identification of deregulated miRNA and protein targets related to the pathogenic profile of neutrophils in rheumatoid arthritis. In silico studies were performed to identify eight altered miRNA using QIAGEN's Ingeunity Pathway Analysis (IPA, QIAGEN Redwood City, USA; $h$ ttps://analysis.ingenuity.com) as the main regulators of proteins involved in the abnormal activation of neutrophils in RA: inflammation, migration and cell survival. By using the tool miRNA target filter of IPA, a network including the selected miRNA and mRNA targets experimentally observed and predicted with high confidence, was generated. miRNA: microRNA; RA: rheumatoid arthritis.

In vitro treatment of active neutrophils purified from RA patients with IFX restored the low levels of the eight selected miRNA while TCZ only up-regulated the expression of miR-148a (Figure 5D). Accordingly, IFX upregulated the expression levels of AGO1. Regarding mRNA targets, IFX reduced the mRNA expression of $V E G F-A$, TNF- $\alpha, I L-1 \beta, I L-8$ and STAT3. Treatment with TCZ also diminished the expression of various of these mRNA targets such as IL-1 $\beta$, IL-8, IL-6R and STAT3 (Figure 5E). In addition, protein release of TNF- $\alpha$, IL- 8 and IL-1 $\beta$ was reversed in RA neutrophils after treatments with both, IFX and TCZ (Figure 5F).

\section{Overexpression of $\mathrm{miR}-126, \mathrm{miR}-148 \mathrm{a}$ and $\mathrm{miR}-223$ in RA neutrophils decreases specific targets involved in inflammation, migration and cell survival}

We selected three downregulated miRNA to evaluate their role in migration, proinflammatory profile and cell survival of RA neutrophils: miR-223 was the most abundant in neutrophils, miR-148a and miR-126 have several potential and demonstrated mRNA targets involved in inflammation. As visible in Figure 6, miR-126, miR-148a and miR-223 overexpression led to a downregulation of their specific mRNA targets: miR-126 overexpression induced a significant downregulation of VEGF-A protein expression (Figure 6A), and miR-223 overexpression promoted a significant decrease in the protein expression of IL-8 and IL-1. Interestingly, miR-223 transfection induced a significant increase of VEGF $\mathrm{mRNA}$ and protein (Figure $6 C)$. On the other hand, miR-148a overexpression reduced gene and protein expression levels of TNF- $\alpha$ (Figure 6B).

\section{DICER downregulation in neutrophils might exacerbate their inflammatory profile}

Using a low number of lentiviral particles, $25 \%$ of DICER expression was inhibited in HL-60 neutrophil-like cells (Figure 6D). This reduction promoted a significant decrease in all the selected miRNA (Figure 6E). Protein levels of a number of cytokines and chemokines were significantly upregulated in neutrophils after DICER downregulation (Figure 6F).

\section{Discussion}

This study reports for the first time the altered miRNA expression profile in neutrophils from RA patients, describing a defect in the miRNA processing machinery responsible for a global low abundance of miRNA, mediated by ACPA and inflammatory mediators, promoting the high inflammatory profile of these cells in RA.

Several miRNA have been shown to be increased in the $\mathrm{PB}$ and in inflamed joints in RA patients, correlated to disease activity and to promoting the production of inflammatory mediators involved in the synovitis. ${ }^{13,14}$ Likewise, 
A

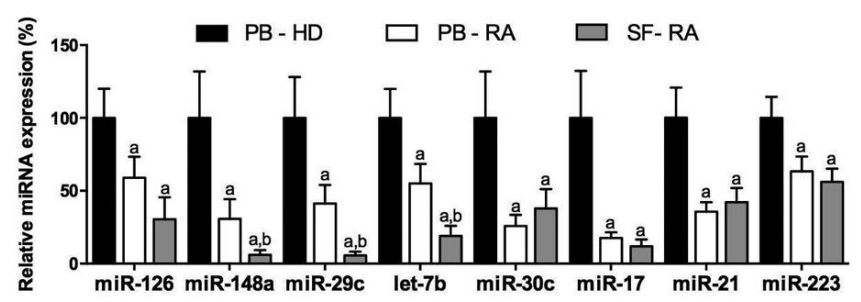

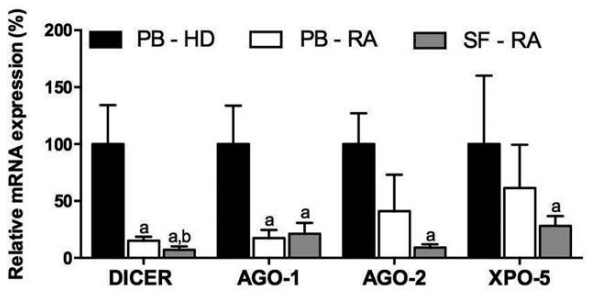

C

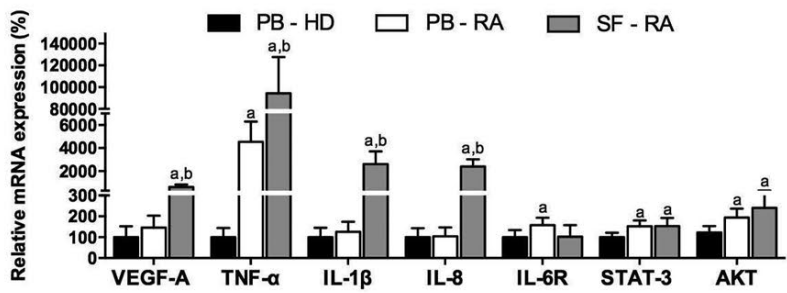

D
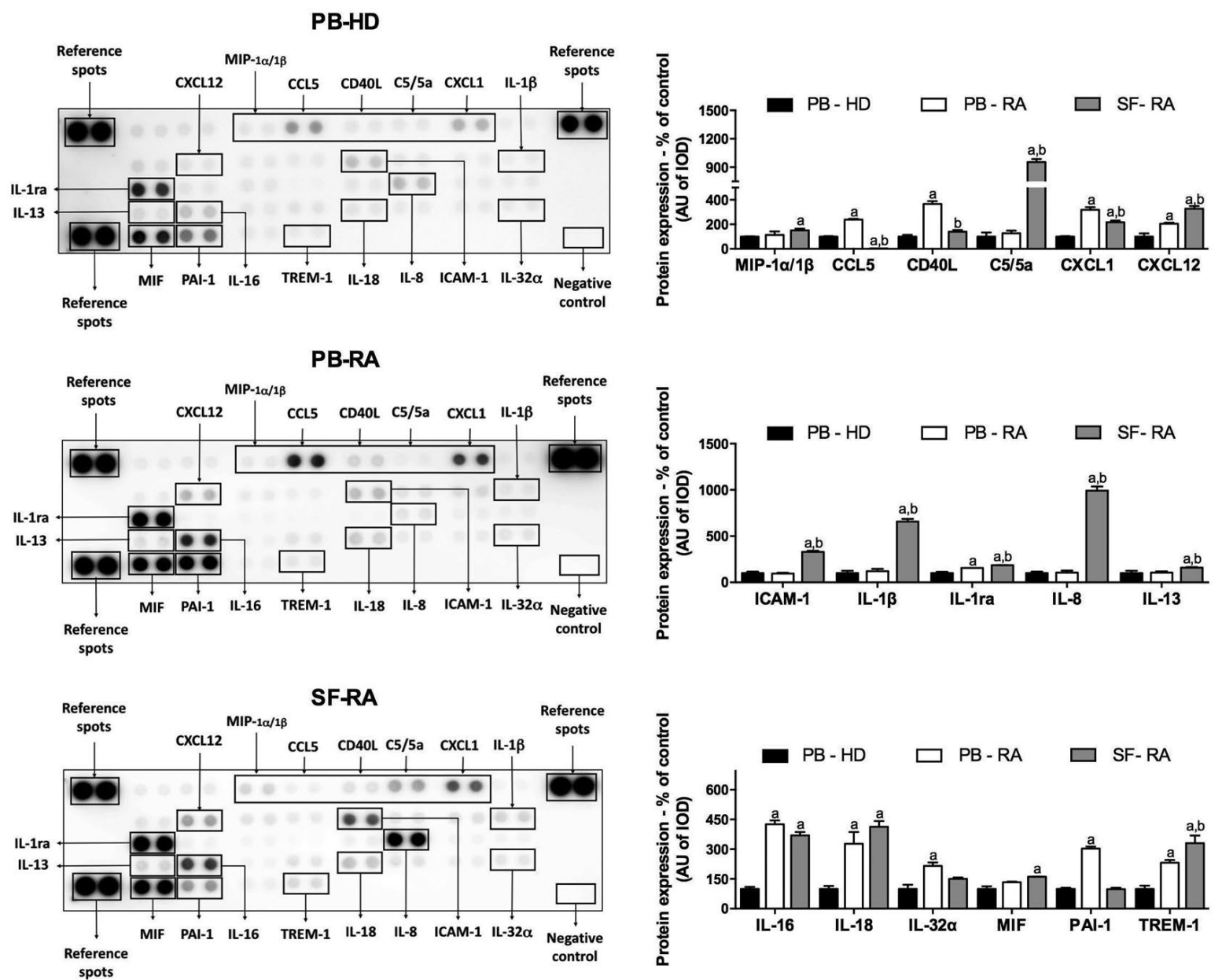

Figure 3. Low abundance of miRNA levels in rheumatoid arthritis neutrophils might be due to a defect in the miRNA processing. (A) Validation of the miRNA array in PB-HD neutrophils $(n=40)$, PB-RA neutrophils $(n=40)$ and SF-RA neutrophils $(n=40)$. (B) Expression of genes related to miRNA biogenesis machinery in PB-HD $(n=40)$, PB-RA $(n=40)$ and SF-RA neutrophils $(n=40)$. (C) Gene expression of putative mRNA targets of the selected miRNA in PB-HD ( $n=40)$, PB-RA $(n=40)$ and SFRA neutrophils $(n=40)$. (D) Proteome profile of chemokines and cytokines in neutrophils from PB-HD $(n=10), P B-R A(n=10)$ and SF-RA neutrophils ( $n=10)$. MiR: microRNA; RA: rheumatoid arthritis; PB: peripheral blood; HD: healthy donor; SF: synovial fluid; AGO-1: argonaute-1; AGO-2: argonaute-2; XPO-5: exportin-5; VEGF-A: vascular endothelial growth factor A; TNF- $\alpha$ : tumor necrosis factor-alpha; IL-1 $\beta$ : interleukin-1 beta; IL-8: interleukin-8; IL-6R: interleukin-6 receptor; STAT-3: signal transducer and activator of transcription 3; AKT: protein kinase B; MIP-1 $\alpha / 1 \beta$ : macrophage inflammatory protein 1 alpha/1 beta; CCL5: chemokine (C-C motif) ligand 5; CD40 ligand: cluster differentiation 40 ligand; C5/5a: complement component C5/5a; CXCL-1: chemokine (C-X-C motif) ligand 1; CXCL12: chemokine (C-X-C motif) ligand 12; ICAM-1: intercellular adhesion molecule 1; IL-1ra: interleukin-1 receptor antagonist; IL-13: interleukin 13; IL-16: interleukin 16; IL-18: interleukin 18; IL-32 $\alpha$ : interleukin 32 alpha; MIF: macrophage migration inhibitory factor; PAI-1: plasminogen activator inhibitor-type 1; TREM-1: triggering receptor expressed on myeloid cells 1 . Data are presented as mean \pm standard error of the mean (SEM); a: significant differences vs. PB-HD P<0.05; b: significant differences vs. PB-RA $P<0.05$. 


\begin{tabular}{|c|c|c|c|c|c|c|c|c|c|}
\hline & miR-126 & miR-148a & miR-29c & let-7c & miR-30c & miR-17 & miR-21 & miR-223 & mRNA DICER \\
\hline ACPA & $\begin{array}{l}r=-0.632 \\
P=0.000\end{array}$ & $\begin{array}{l}\mathrm{r}=-0.566 \\
P=0.000\end{array}$ & $\begin{array}{l}r=-0.575 \\
P=0.000\end{array}$ & $\begin{array}{l}r=-0.656 \\
P=0.000\end{array}$ & $\begin{array}{l}r=-0.372 \\
P=0.023\end{array}$ & $\begin{array}{l}r=-0.263 \\
P=0.071\end{array}$ & $\begin{array}{l}r=-0.369 \\
P=0.032\end{array}$ & $\begin{array}{l}r=-0.344 \\
P=0.050\end{array}$ & $\begin{array}{l}r=-0.537 \\
P=0.005\end{array}$ \\
\hline DAS28 & $\begin{array}{l}r=-0.467 \\
P=0.005\end{array}$ & $\begin{array}{l}r=-0.455 \\
P=0.004\end{array}$ & $\begin{array}{l}r=-0.446 \\
P=0.006\end{array}$ & $\begin{array}{l}r=-0.512 \\
P=0.002\end{array}$ & $\begin{array}{l}r=-0.348 \\
P=0.028\end{array}$ & $\begin{array}{l}r=-0.136 \\
P=0.346\end{array}$ & $\begin{array}{l}r=-0.326 \\
P=0.046\end{array}$ & $\begin{array}{l}r=-0.385 \\
P=0.050\end{array}$ & $\begin{array}{l}\mathrm{r}=-0.538 \\
P=0.071\end{array}$ \\
\hline CRP & $\begin{array}{l}r=-0.486 \\
P=0.004\end{array}$ & $\begin{array}{l}r=-0.404 \\
P=0.015\end{array}$ & $\begin{array}{l}r=-0.438 \\
P=0.008\end{array}$ & $\begin{array}{l}r=-0.480 \\
P=0.005\end{array}$ & $\begin{array}{l}r=-0.141 \\
P=0.344\end{array}$ & $\begin{array}{l}r=-0.177 \\
P=0.225\end{array}$ & $\begin{array}{l}r=-0.512 \\
P=0.002\end{array}$ & $\begin{array}{l}r=-0.388 \\
P=0.067\end{array}$ & $\begin{array}{l}\mathrm{r}=-0.552 \\
P=0.063\end{array}$ \\
\hline Serum TNF- $\alpha$ & $\begin{array}{l}r=-0.518 \\
P=0.125\end{array}$ & $\begin{array}{l}r=0.035 \\
P=0.440\end{array}$ & $\begin{array}{l}r=0.005 \\
P=0.973\end{array}$ & $\begin{array}{l}r=0.023 \\
P=0.440\end{array}$ & $\begin{array}{l}r=0.178 \\
P=0.440\end{array}$ & $\begin{array}{l}r=0.178 \\
P=0.440\end{array}$ & $\begin{array}{l}r=-0.530 \\
P=0.115\end{array}$ & $\begin{array}{l}\mathrm{r}=0.178 \\
P=0.440\end{array}$ & $\begin{array}{l}r=-0.496 \\
P=0.048\end{array}$ \\
\hline Serum IL-6 & $\begin{array}{l}r=-0.117 \\
P=0.439\end{array}$ & $\begin{array}{l}r=0.035 \\
P=0.815\end{array}$ & $\begin{array}{l}r=0.005 \\
P=0.973\end{array}$ & $\begin{array}{l}r=0.023 \\
P=0.880\end{array}$ & $\begin{array}{l}r=-0.006 \\
P=0.964\end{array}$ & $\begin{array}{l}r=-0.082 \\
P=0.569\end{array}$ & $\begin{array}{l}\mathrm{r}=-0.042 \\
P=0.779\end{array}$ & $\begin{array}{l}r=-0.182 \\
P=0.335\end{array}$ & $\begin{array}{l}r=-0.266 \\
P=0.148\end{array}$ \\
\hline
\end{tabular}

B

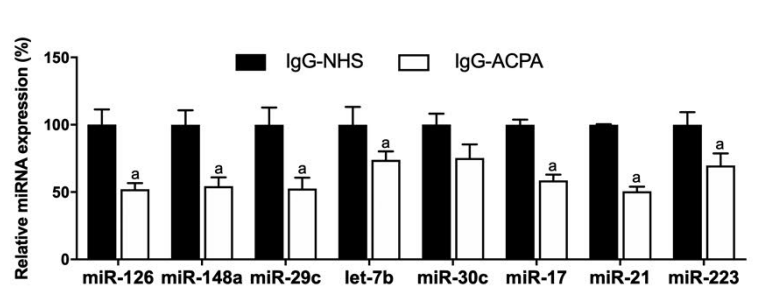

D

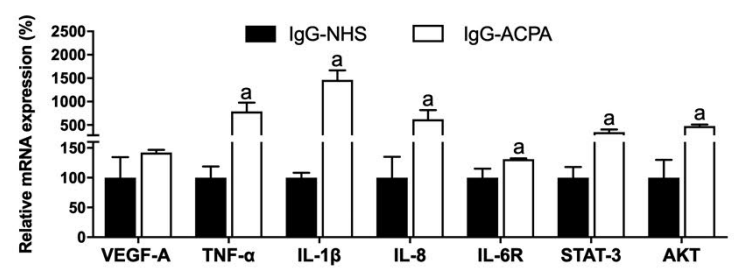

IgG-NHS

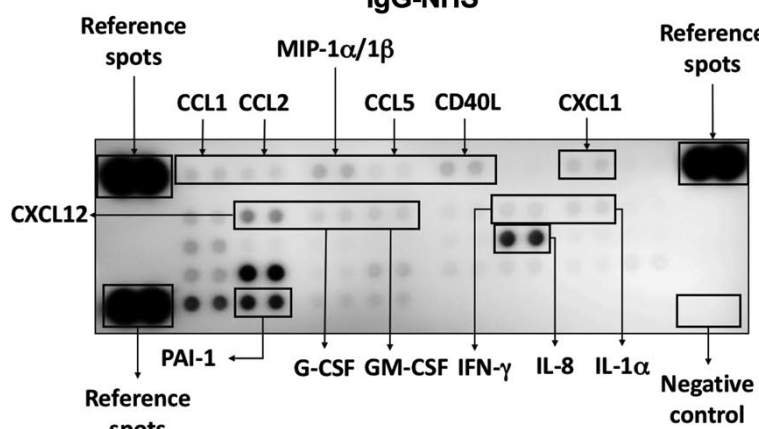

IgG-ACPA

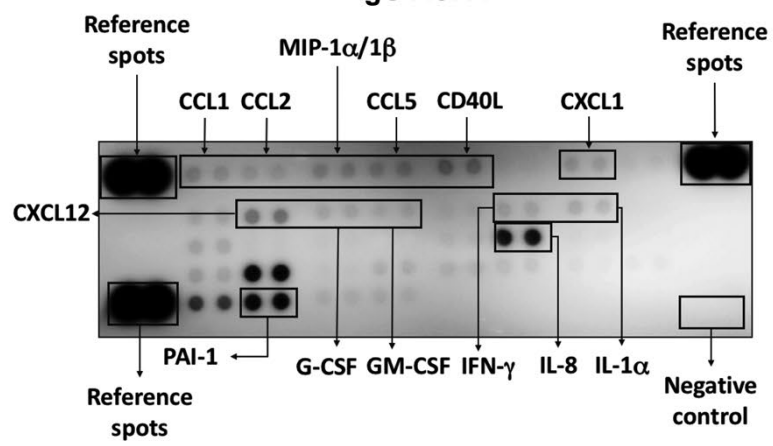

C

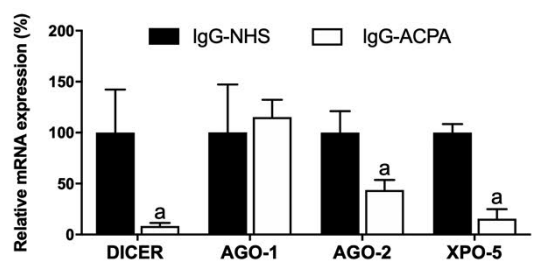

E

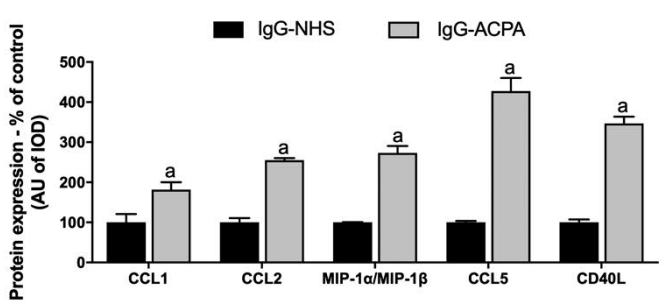

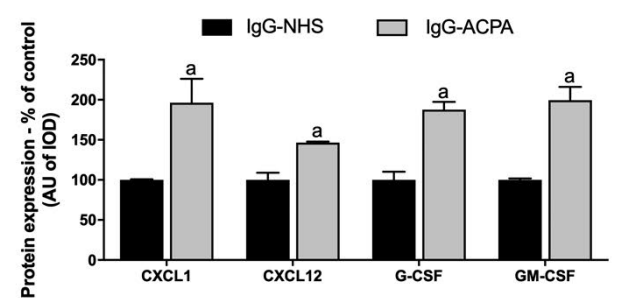

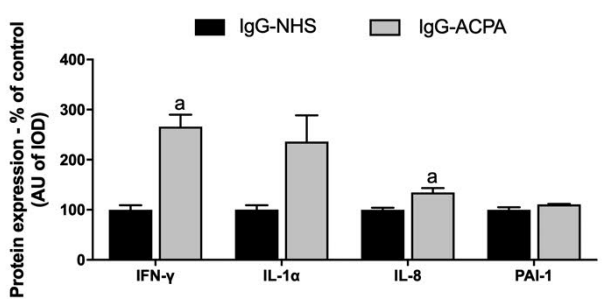

Figure 4. Reduced levels of miRNA in rheumatoid arthritis neutrophils are related to ACPA. (A) Correlation studies of reduced miRNA and DICER expression levels in neutrophils from peripheral blood with clinical parameters such as, ACPA levels, DAS28, CRP and inflammatory markers including TNF- $\alpha$ and IL-6 serum cytokine levels. (B) In vitro effect of enriched IgG-ACPA in miRNA expression in healthy neutrophils. (C) In vitro effect of enriched IgG-ACPA in the expression of genes related to the miRNA biogenesis in healthy neutrophils. (D) In vitro effect of enriched IgG-ACPA in the mRNA expression of potential mRNA targets of the validated miRNA. (E) Proteome profile of chemokines and cytokines in neutrophils treated with log-NHS and enriched log-ACPA. ACPA: antibodies to citrullinated protein antigens; IgGNHS: immung ne: 1 1 beta, IL-8: interle ligand 1; CCL2: chemokine (C-C motif) ligand 2, MIP-1 $\alpha / 1 \beta$ : macrophage inflammatory protein 1 alpha/ 1 beta; CCL5: chemokine (C-C motif) ligand 5 ; $C D 40$ ligand: cluster differentiation 40 ligand; CXCL-1: chemokine (C-X-C motif) ligand 1; CXCL12: chemokine (C-X-C motif) ligand 12; G-CSF: granulocyte colony-stimulating factor; GM-CSF: granulocyte-macrophage colony-stimulating factor; IFN- $\gamma$ : interferon gamma; IL-1 $\alpha$ : interleukin-1 alpha; PAI-1: plasminogen activator inhibitor-type 1. Data are presented as mean \pm standard error of the mean (SEM) $(n=5)$; a: significant differences $P<0.05$. 
A
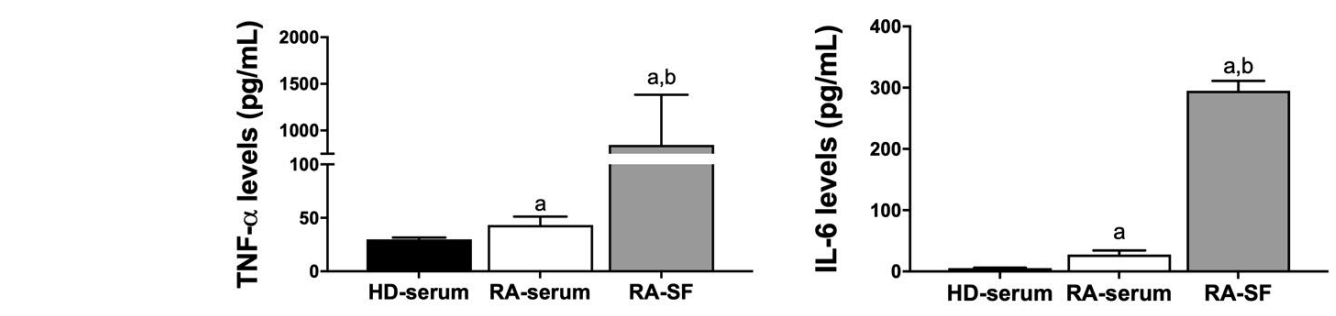

B

HD-serum RA-serum RA-SF
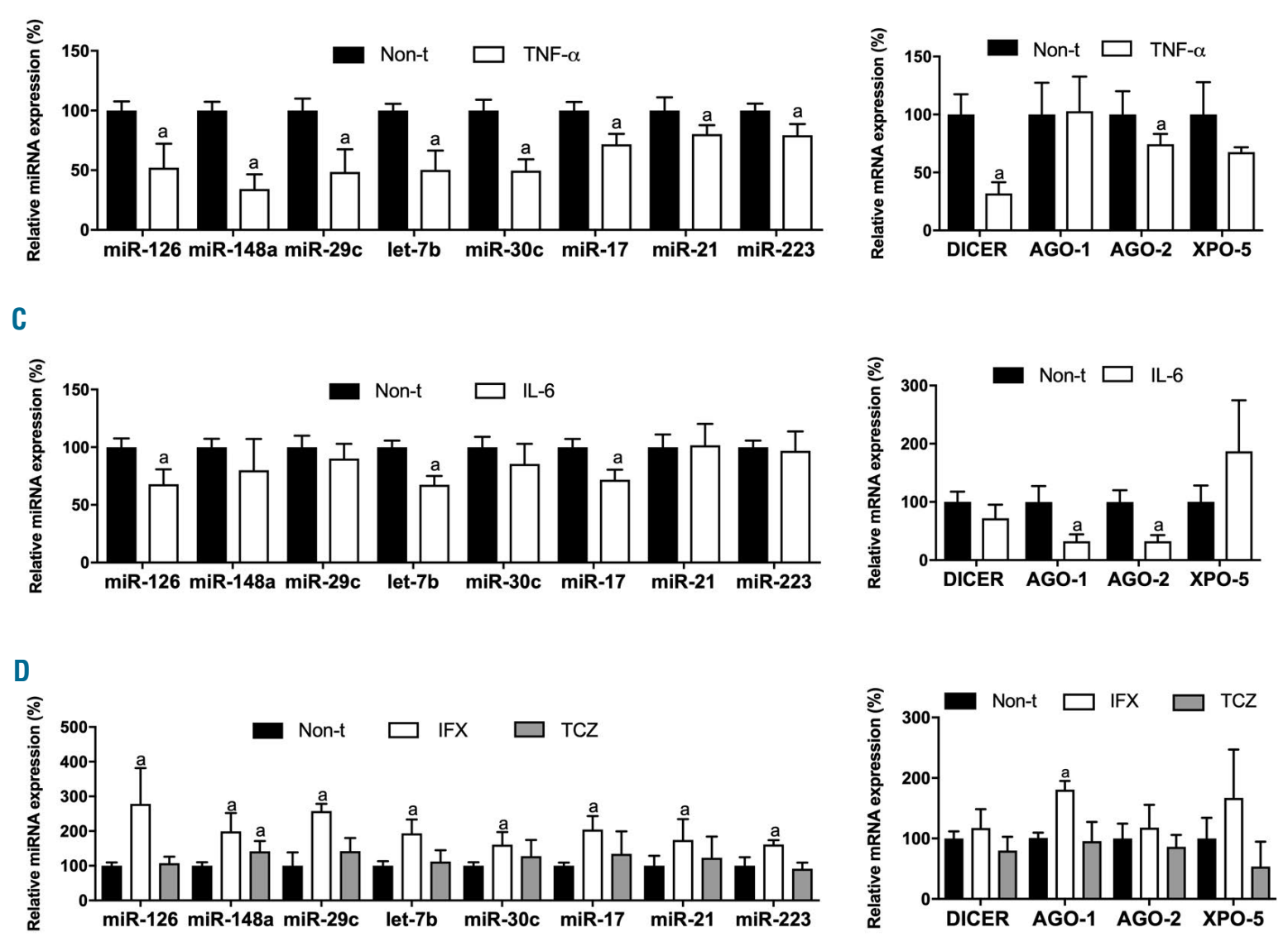

E

$\mathbf{F}$
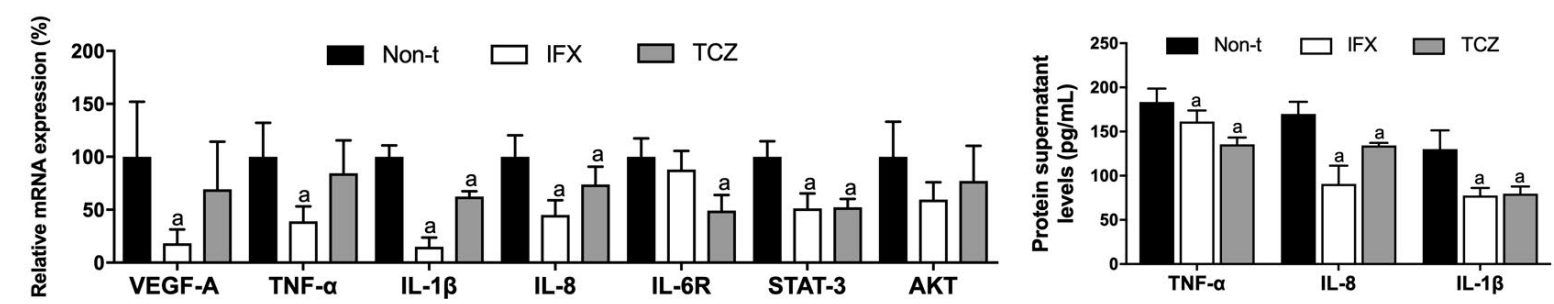

Figure 5. Modulation of the expression of miRNA and genes involved in their processing in healthy donor neutrophils by inflammatory components - in vitro effects of TCZ and IFX in RA active neutrophils. (A) Serum and synovial levels of TNF- $\alpha$ and IL-6 (40 HD-serum, 40 RA-serum and 40 RA-SF). a: significant differences vs. HD serum $P<0.05$; b: significant differences vs. RA serum $P<0.05$. (B) Expression of miRNA and genes involved in their biogenesis machinery in HD neutrophils treated with TNF- $\alpha$. (C) Expression of miRNA and genes involved in their biogenesis machinery in HD neutrophils treated with IL-6. (D) Expression of miRNA and genes involved in their processing in RA active neutrophils treated in vitro with TCZ or IFX. (E) Gene expression of putative mRNA targets of the validated miRNA in RA active neutrophils treated in vitro with TCZ or IFX. (F) Protein levels of putative mRNA targets of the validated miRNA in RA active neutrophils treated in vitro with TCZ or IFX. miRNA: microRNA; HD: healthy donor; TCZ: tocilizumab; IFX: infliximab; RA: rheumatoid arthritis; AGO-1: argonaute-1; AGO-2: argonaute-2; XPO-5: exportin-5; VEGFA: vascular endothelial growth factor; TNF- $\alpha$ : tumor necrosis factor-alpha; IL-1 $\beta$ : interleukin-1 beta; IL-8: interleukin-8; IL-6R: interleukin-6 receptor; STAT-3: signal transducer and activator of transcription 3; AKT: protein kinase B; Non-t: non-treated. Data are presented as mean \pm standard error of the mean (SEM) of five independent experiments; a: significant differences respective non-treated $P<0.05$. 
A

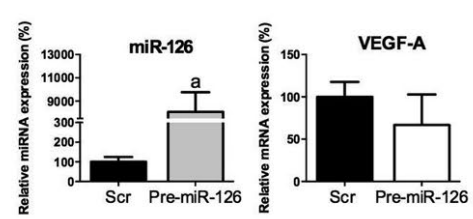

B

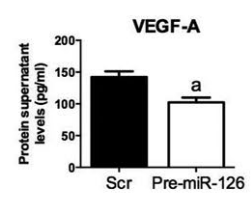

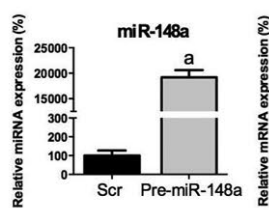
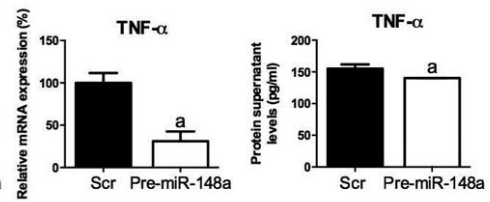

C

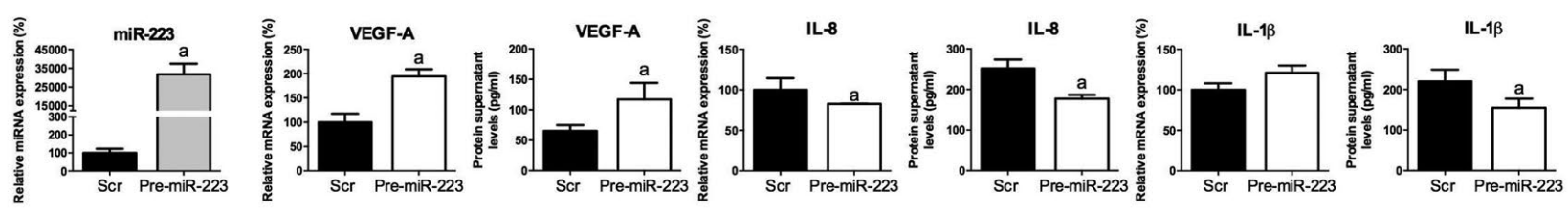

D

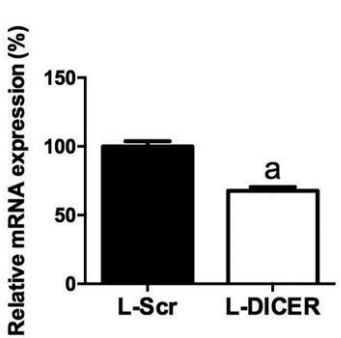

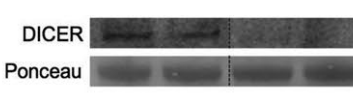

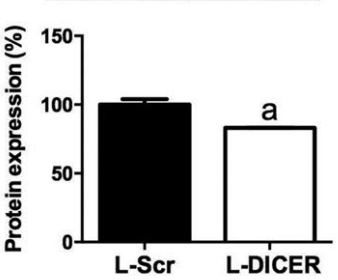

$255 \mathrm{kDa}$

E

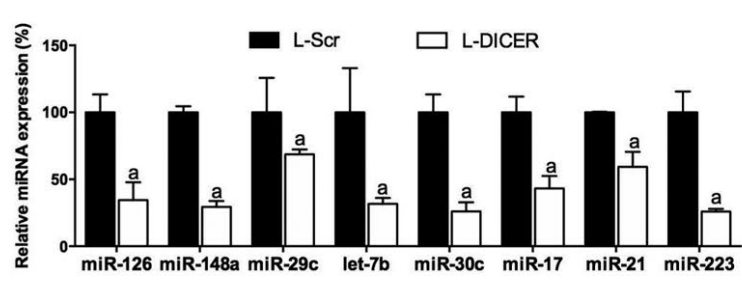

F

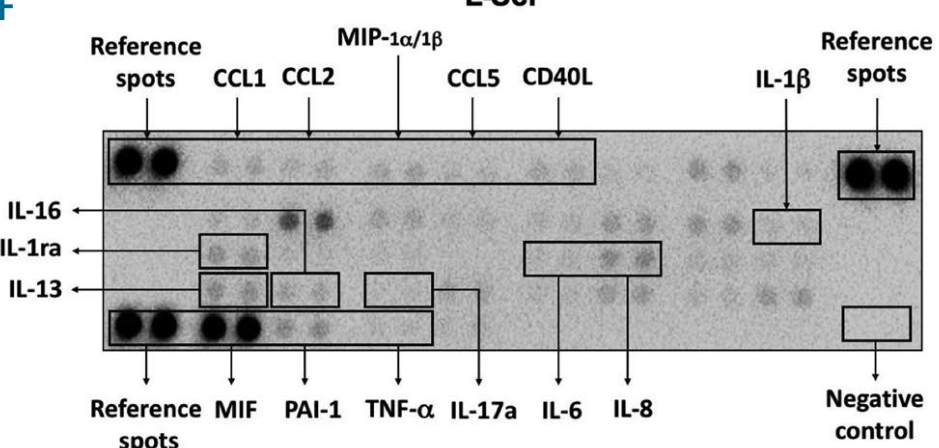

\section{L-DICER}

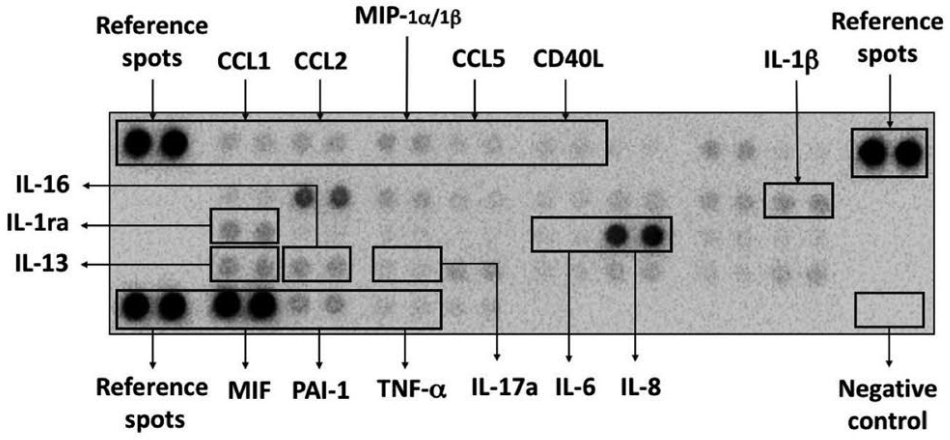

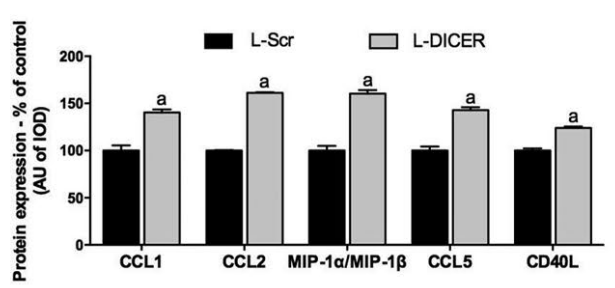
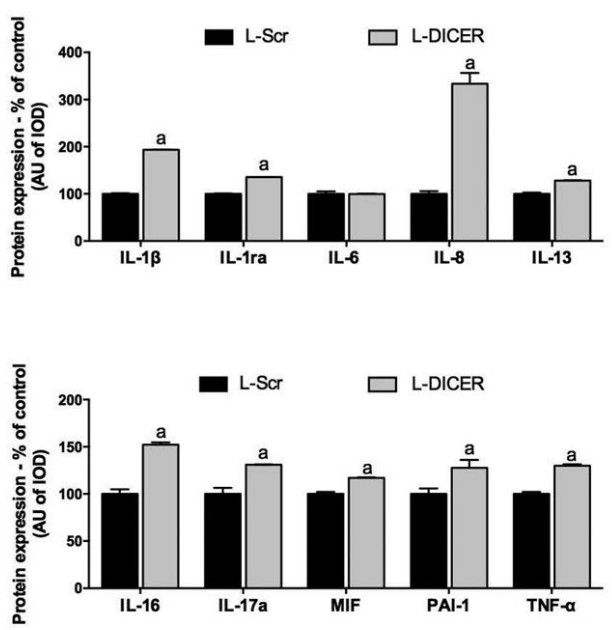

Figure 6. miR-126, miR-148a and miR-223 regulate the expression of specific mRNA targets involved in inflammation, migration and cell survival on neutrophils. DICER downregulation induces an inflammatory profile in neutrophils. (A) Overexpression of miR-126 decreased the expression of VEGF in RA active neutrophils. (B) Overexpression of miR-148a decreased the expression of TNF- $\alpha$ in RA active neutrophils. (C) Overexpression of miR-223 decreased the expression of IL-8 and II-1 $\beta$ and increased VEGF in RA active neutrophils. (D) DICER expression levels after lentivirus transfection by PCR and Western blot. (E) Expression levels of miRNA in DICER downregulated neutrophils. (G) Protesome array of cytokines and chemokines in HL60 neutrophil-like cells after downregulation of DICER by lentiviral transfection. MiRNA: microRNA; RA: rheumatoid arthritis; VEGF-A: vascular endothelial growth factor A; TNF- $\alpha$ : tumor necrosis factor-alpha; IL-8: interleukin-8; IL-1 $\beta$ : Interleukin-1 beta; CCL1: chemokine (C-C motif) ligand 1; CCL2: chemokine (C-C motif) ligand 2; MIP-1 $\alpha / 1 \beta$ : macrophage inflammatory proteins alpha and beta; CCL5: chemokine (C-C motif) ligand 5; IL-1ra: interleukin 1 receptor antagonist; IL-6: interleukin-6; IL-13: interleukin-13; IL-16: interleukin-16; IL-17a: interleukin-17a; MIP: macrophage migration inhibitory factor; PAI-1: plasminogen activator inhibitor-1; Scr: scrambled; L-Scr: lentivirus-Scrambled; L-DICER: lentivirus-DICER. Data are presented as mean \pm standard error of the mean (SEM) of two independent experiments; a: significant differences $P<0.05$. 
various single nucleotide polymorphism have been studied in miRNA in RA. ${ }^{15}$

Our study shows a global downregulation of the miRNA expression in RA neutrophils, more marked in SF neutrophils, suggesting that it might contribute to the abnormal activated profile of these cells in the synovium.

In this sense, global downregulation of miRNA has been shown in human alveolar macrophages induced by cigarette smoking, responsible for the changes in gene expression associated with the disease. ${ }^{16}$ In autoimmune disorders, we recently described a global downregulation of the miRNA levels in neutrophils from patients with systemic lupus erythematosus and antiphospholipid syndrome, which may indicate that chronic inflammation and/or autoimmunity is associated with a reduction of miRNA in neutrophils. ${ }^{17}$ In the present study, we demonstrate that either ACPA or inflammatory mediators, especially TNF- $\alpha$ can modulate the miRNA expression profile, through a reduction of several proteins involved in its processing, which might be translated into an increase of genes that might be involved in inflammation, cell survival and migration.

Up to date, no study has reported the effect of ACPA on the expression of miRNA. In our hands, the reduced levels of miRNA and DICER in RA neutrophils correlated with elevated levels of ACPA. Accordingly, our in vitro studies demonstrated a direct involvement of these autoantibodies in the deregulation of various miRNA and their specific protein targets- globally related to the pathogenesis of RA. We further demonstrated that the global downregulation of the miRNA expression in RA neutrophils was associated, at least partially, with the reduced levels of DICER. A recent study suggested the role of DICER in neutrophils differentiation, where the DICER inhibition attenuated the activation of autophagy, a process that is needed for proper neutrophil differentiation. ${ }^{12}$ DICER plays a crucial role in miRNA biogenesis. Thus, it has been suggested that mRNA and protein levels of DICER must be strictly controlled since small changes can initiate various pathological processes. ${ }^{18}$ Here, we prove a novel role for DICER in neutrophils, showing that a small reduction of protein levels can induce a proinflammatory profile in neutrophils by downregulating several miRNA and, hence, a number of putative targeted cytokines and chemokines.

Currently, little is known about the miRNA regulating neutrophil function. Several miRNA have recently been involved in the neutrophil development and function and in various pathological states, including miRNA-155, miRNA-34a, miRNA-223, miRNA-142, miRNA-452 and miRNA-466L. ${ }^{19}$ Overexpression of miRNA-155 and miRNA-34a in neutrophils from patients with myelodysplastic syndrome has been shown to contribute to an alteration of the migration. ${ }^{20}$ In addition, decreased levels of both, the miRNA-145 and the miRNA-143 have been shown in acute myelogenous leukemia, which are responsible for the blockade of the differentiation process of the neutrophils. ${ }^{21}$

Alongside with previous evidence, here we show that miRNA-223 is one of the most abundant miRNA on neutrophils. ${ }^{22}$ It has recently been demonstrated that the miRNA-223 is an important regulator blocking the infiltration of neutrophils in alcoholic hepatic disease. ${ }^{22}$ Supporting a role for this miRNA in the infiltration capacity of the neutrophils, in the present work we demon- strated how the overexpression of the miRNA-223 in neutrophils of RA patients reduced specifically the expression of IL-1 and IL-8, molecules involved in inflammation and migration.

The role of the miRNA-126 in the vascular integrity has been also evidenced. ${ }^{23}$ We observed reduced levels of the miRNA-126 in RA neutrophils, while its induced overexpression in RA neutrophils significantly reduced the levels of VEGF, pointing to the role of this miRNA in neutrophil adhesion and migration.

Multiple functions have been attributed to miRNA$148 \mathrm{a}$ in several diseases and low levels of miRNA-148 have been shown to be related to less survival time and increased recurrence risk in bladder cancer. ${ }^{24}$ In addition, miRNA-148 has been related to innate and adaptive immune responses. ${ }^{25}$ Our data is in agreement with these studies, since we found reduced levels of the miRNA-148 in RA neutrophils, associated to increased levels of TNF, a key inflammatory protein driving the RA disease.

Other miRNA found decreased in RA neutrophils, such as miRNA-21, Let-7 and miRNA-30, have previously been reported to be altered in different types of tumors, thus playing a relevant role in tumorigenesis, invasion and metastasis of cancer cells. ${ }^{26-29}$ In addition, Let-7 and miRNA-17 regulate the T-cell response..$^{27,30}$ Finally, a recent study demonstrated that the levels of all the members of the miRNA ${ }^{29}$ family were decreased in $\mathrm{PB}$ mononuclear cells and $\mathrm{CB} 34^{+}$cells of the bone marrow of acute myelogenous leukemia patients. The normalization of their levels partially inhibited the abnormal proliferation of the blasts, blocked the myeloid differentiation and repressed the apoptosis. ${ }^{31}$

TNF- $\alpha$ and IL- 6 are key inflammatory effectors in RA, whose levels are elevated in RA serum and further increased in RA SF. We found a marked effect of TNF- $\alpha$ on neutrophils, lowering the expression of genes related to miRNA processing (including DICER and AGO-1) and downregulating the eight miRNA selected. By contrast, IL- 6 had less effect but was still able to reduce the levels of miRNA-126, let-7b and miRNA-17 alongside with the expression of AGO-1 and AGO-2. Treatment of active RA neutrophils with IFX or TCZ reduced the inflammatory profile, by downregulating the gene expression of VEGFA, TNF- $\alpha, I L-1 \beta, I L 8, I L 6 R$ and STAT3. However, only IFX was able to restore the global levels of selected miRNA, alongside with genes involved in their processing in RA neutrophils, an effect that might be expected after the efficciency of TNF- $\alpha$ observed to reduce the miRNA levels, thus suggesting that IFX might specifically minimize the abnormal profile of the RA neutrophils through the inhibition of TNF- $\alpha$, which directly acts by reducing the expression of miRNA. In agreement with these results, we recently demonstrated that in vivo treatment with anti-TNF- $\alpha$ drugs for six months regulated the levels of several miRNA in the plasma of RA patients. Moreover, miRNA-23 and miRNA-223 were identified as potential biomarkers of therapy effectiveness. ${ }^{32}$

Altogether, our study shows that neutrophils from RA patients have a defect in the miRNA biogenesis machinery, are more marked in SF neutrophils, and are induced by ACPA and inflammatory mediators. This defect might be directly associated with the abnormal neutrophil activation, thus increasing their proinflammatory profile, observable by the higher expression of a number of chemokines and cytokines. 
Among the miRNA altered in RA neutrophils, we demonstrate that the miRNA-223, miRNA-126 and miRNA-148 are involved in the modulation of genes involved in processes such as migration, inflammation and cell survival in neutrophils. Finally, biological therapies would be able to improve miRNA processing, upregulating the levels of miRNA, which might reduce the activation of the neutrophil.

Beyond the regulation of miRNA in RA neutrophils, there are likely other epigenetic mechanisms that potentially contribute to the abnormal activation of these cells in RA context, such as chromatin modification.

This study has several limitations: it is a cross-sectional study in which consecutive patients from standard clinical practice were recruited. These patients were treated with standard therapy, including immunosuppressants, at the time of the sample and clinical detail collection. Thus, the effects of specific treatments on the expression levels of miRNA or the molecules involved in their biogenesis in neutrophils was not analyzed.

The isolation of neutrophils with anti-CD15 microbeads is a further potential limitation of this work, however, up to date no unique method for the isolation of neutrophil isolation has been accepted worldwide and differing techniques have shown either some activation or functional impairment of the cells and the presence of small amounts of contaminating cells.

Choosing an adequate method to isolate neutrophils from SF is thus challenging. In our hands, after testing a variety of potential priming/ activation techniques and the percentage of contaminating cells, the isolation of neutrophils with anti-CD15 microbeads was chosen as a suitable approach to obtain sufficiently numbers of inactivated neutrophils. Nevertheless, a consensus on the selection of the right isolation method that allows the comparison of neutrophil paired samples from SF and $\mathrm{PB}$ is still needed.

\section{Acknowledgments}

We thank all the patients for their kind participation in this study.

\section{Funding}

This work was supported by grants from the Instituto de Salud Carlos III (CP15/00158, PI17/01316 and PI18/00837), cofinanciado por el fondo europeo de desarrollo regional de la Union Europea, 'una manera de hacer Europa', Spain, the Regional Health System (ref. PIO285 2017), and the Spanish Inflammatory and Rheumatic Diseases Network (RIER, RD16/0012/0015). CL-P was supported by contracts from both the Junta de Andalucia and Spanish Ministry of Science and Innovation (Ramon y Cajal). NB was supported by Ministry of Health postdoctoral fellowship (Miguel Servet Programme). YJ-G was supported by a contract from the University of Cordoba [co-funded by the Research Plan of the University of Cordoba and the Operating Program of the European Regional Development Funds (ERDF) for Andalusia. CL-P was supported by a contract from the Junta de Andalucia] (Nicolas Monardes Programme). YJ-G was supported by a contract from the University of Cordoba (co-funded by the Research Plan of the University of Cordoba and the Operating Program of the European Regional Development Funds [ERDF]) for Andalusia).

\section{References}

1. Deng GM, Lenardo $M$. The role of immune cells and cytokines in the pathogenesis of rheumatoid arthritis. Drug Discovery Today: Disease Mechanisms. 2006; 3(2):163-168.

2. Barbarroja N, Pérez-Sanchez C, RuizLimón P, et al. Anticyclic citrullinated protein antibodies are implicated in the development of cardiovascular disease in rheumatoid arthritis. Arterioscler Thromb Vasc Biol. 2014;34(12):2706-2716.

3. Song, YW, Kang EH. Autoantibodies in rheumatoid arthritis: rheumatoid factors and anticitrullinated protein antibodies. QJM. 2010;103(3):139-146.

4. Corsiero E, Pratesi F, Prediletto E, Bombardieri M, Migliorini P. NETosis as source of autoantigens in rheumatoid arthritis. Front Immunol. 2016;7:485.

5. Araki, Y., and Mimura, T. The mechanisms underlying chronic inflammation in rheumatoid arthritis from the perspective of the epigenetic landscape. J Immunol Res. 2016;2016:6290682.

6. Ceribelli A, Yao B, Dominguez-Gutierrez PR, Nahid MA, Satoh M, Chan EK. MicroRNAs in systemic rheumatic diseases. Arthritis Res Ther. 2011;13(4):229.

7. Fabian MR, Sonenberg N, Filipowicz W. Regulation of mRNA translation and stability by microRNAs. Ann Rev Biochem. 2010;79:351-379.
8. Chendrimada TP, Gregory RI, Kumaraswamy E. TRBP recruits the Dicer complex to Ago2 for microRNA processing and gene silencing. Nature. 2005; 436(7051):740-744

9. Gregory RI, Chendrimada TP, Cooch N, Shiekhattar R. Human RISC couples microRNA biogenesis and posttranscriptional gene silencing. Cell. 2005;123(4):631640

10. Bartel DP. MicroRNAs: target recognition and regulatory functions. Cell. 2009; 136(2):215-233.

11. Rajasekhar M, Olsson AM, Steel KJ, et al. MicroRNA-155 contributes to enhanced resistance to apoptosis in monocytes from patients with rheumatoid arthritis. J Autoimmun. 2017;79:53-62.

12. Zahler S, Kowalski C, Brosig A, Kuppat C, Becker BF, Gerlach, E. The function of neutrophils isolated by a magnetic antibody cell separation technique is not altered in comparison to a density centrifugation method. J Immunol Methods. 1997;200(12):173-179.

13. Duroux-Richard I, Jorgensen C, Apparailly F. What do microRNAs mean for rheumatoid arthritis? Arthritis Rheum. 2011; 64(1):11-20.

14. Kurowska-Stolarska M, Alivernini S, Ballantine L, et al. MicroRNA-155 as a proinflammatory regulator in clinical and experimental arthritis. Proc Natl Acad Sci U S A. 2011:108(27):11193-11198.

15. Yang B, Zhang JL, Shi YY, et al. Association study of single nucleotide polymorphisms in pre-miRNA and rheumatoid arthritis in a Han Chinese population. Mol Biol Rep. 2011;38(8):4913-4919.

16. Graff JW, Powers LS, Dickson AM, et al. Cigarette smoking decreases global microRNA expression in human alveolar macrophages. PLoS One. 2012;7(8):e44066.

17. Pérez-Sánchez C, Aguirre MA, Ruiz-Limón $\mathrm{P}$, et al. Atherothrombosis-associated microRNAs in Antiphospholipid syndrome and Systemic Lupus Erythematosus patients. Sci Rep. 2016;6:31375.

18. Kurzynska-Kokorniak A, Koralewska N, Pokornorwska M, et al. The many faces of Dicer: the complexity of the mechanisms regulating Dicer gene expresssion and enzyme activities. Nucleic Acids Res. 2015; 43(9):4365-4380

19. Gurol T, Zhou W, Deng Q. MicroRNAs in neutrophils: potential next generation therapeutics for inflammatory ailments. Immunol Rev. 2016;273(1):29-47.

20. Cao M, Shikama $Y$, Kimura $H$, et al. Mechanisms of impaired neutrophil migration by microRNAs in myelodysplastic syndromes. J Immunol. 2017;198(5):1887-1899.

21. Batliner J, Buehrer E, Fey M, and Tschan $M$ Inhibition of the miR-143/145 cluster attenuated neutrophil differentiation of APL cells. Leuk Res. 2012;36(2):237-240.

22. Li M, He Y, Zhou Z, et al. MicroRNA-223 ameliorates alcoholic liver injury by inhibiting the IL-6-p47 oxidative stress pathway in neutrophils. Gut. 2017;66(4):705-715. 
23. Chen H, Li L, Wang S, et al. Reduced miRNA-126 expression facilitates angiogenesis of gastric cancer through its regulation on VEGF-A. Oncotarget. 2014;5(23): 11873-85.

24. Miao C, Zhang J, Zhao K, et al. The significance of microRNA-148/152 family as a prognostic factor in multiple human malignancies: a meta-analysis. Oncotarget. 2017; 8(26):43344-43355

25. Chen Y, Song YX, Wang ZN. The microRNA-148/152 family: multi-faceted players. Mol Cancer. 2013;12:43.

26. Canfrán-Duque A, Rotllan N, Zhang X, et al. Macrophage deficiency of miR-21 promotes apoptosis, plaque necrosis, and vas- cular inflammation during atherogenesis. EMBO Mol Med. 2017;9(9):1244-1262

27. Wells AC, Daniels KA, Angelou CC, et al Modulation of let-7 miRNAs controls the differentiation of effector CD8 T cells. Elife. 2017;6.

28. Liu Y, Zhou Y, Gong X, Zhang C. MicroRNA-30a-5p inhibits the proliferation and invasion of gastric cancer cells by targeting insulin-like growth factor 1 receptor. Exp Ther Med. 2017;14(1);173-180.

29. $\mathrm{Hu} \mathrm{H}$, Li H, He Y. MicroRNA-17 downregulates expression of the PTEN gene to promote the occurrence and development of adenomyosis. Exp Ther Med. 2017; 14(4):3805-3811.
30. Nandakumar P, Tin A, Grove ML, et al MicroRNAs in the miR-17 and miR-15 families are downregulated in chronic kidney disease with hypertension. PLoS One. 2017;12(8):e0176734.

31. Gong J, Yu J, Lin $\mathrm{H}$, et al. The role, mechanism and potentially therapeutic application of microRNA-29 family in acute myeloid leukemia. Cell Death Differ. 2013; 21(1):100-112.

32. Castro-Villegas C, Pérez-Sánchez C, Escudero A, et al. Circulating miRNAs as potential biomarkers of therapy effectiveness in rheumatoid arthritis patients treated with anti-TNFo. Arthritis Res Ther. 2015; $17: 49$. 